\title{
Diffusion Processes via Parabolic Equations: An Infinitesimal approach to Lindeberg's limit theorem.
}

\author{
HEINZ WEISSHAUPT ${ }^{1,2,3}$
}

\begin{abstract}
We approach infinitesimal diffusion processes via a linkage to the diffusion equation. By this we obtain Lindeberg's limit theorem and a Lindeberg type limit theorem for diffusion processes by an application of the underspill principle.

2000 Mathematics Subject Classification 26E35 (primary); 60F05, 60J60, 35K99, $03 \mathrm{H} 05$ (secondary)

Keywords: nonstandard analysis, diffusion processes, infinitesimal models of white noise, Lindeberg type theorems
\end{abstract}

\section{Introduction}

The evolution of diffusions is in standard mathematics described either by ordinary stochastic differential equations, or by partial differential equations, called diffusion equations. Both descriptions are usually connected via the Ito formula. (See [20] Section 5.2, [31] Sections 5.1-5.3, [28] Section 7.3, [18] Theorem 4.8.6., [32] Section 6, [14] Section 1.8 and [16] Section 5.1.)

Nonstandard diffusion theory is usually approached by linking the nonstandard stochastic process defined on a near interval by the concept of Loeb measure [25] to a corresponding standard stochastic process, as described in [8] or [35]. This approach to diffusion theory started with a nonstandard construction of the Brownian motion and the Ito integral in [1] and was further extended to stochastic integration in a broader context in [22] and applied to the analysis of the Ornstein-Uhlenbeck Process [23]

\footnotetext{
${ }^{1}$ Research has been partially supported by a research scholarship of the University of Vienna, Austria

${ }^{2}$ I would like to thank the organizers of the international congress NSM2006 "Nonstandard Methods and Applications in Mathematics" held in Pisa, May 25-31, 2006 for supporting my participation in the conference, including a talk on the topic of this article.

${ }^{3}$ The preparation of the final version of this article was supported by the BMBF, Germany, trough FRISYS (Freiburg Initiative for Systems biology), Kennzeichen 0313921.
} 
and the Malliavin Calculus [9] (see also [24]). Some further important developments concerning Loeb measures on Hyperfinite spaces can be found in [12]. Their abstraction leads to the concept of neocompactness that can be used to prove the existence of solutions of stochastic differential equations with special properties [17].

Another way of linking nonstandard stochastic processes to the standard mathematical world - by Nelson's reduction algorithm - is described in [27]. A third possibility [3] is to use hyper-finite combinatorics and the concept of equivalent processes ([27] Chapter 17) together with path-wise versions of Ito's formula and Girsanov's theorem.

We approach diffusions via infinitesimal stochastic difference equations and call the solutions of these equations infinitesimal diffusion processes. To connect the infinitesimal diffusion processes to the world of standard mathematics we employ Kolmogorov's backward equation.

By this connection we further prove that the standard parts of the expectations of the infinitesimal diffusion processes are independent of the choice of the underlying infinitesimal model of white noise.

This fact can be interpreted as an infinitesimal version of a Lindeberg type limit theorem. By the underspill-principle of nonstandard analysis this enables us to prove a Lindeberg type limit theorem for diffusions in standard mathematical terms. This limit theorem is contrasted by various approximation theorems provided in [18].

Note that the relation between nonstandard diffusion processes and the diffusion equation has - in a different way - already been investigated in [4]. Another, similar connection between a stochastic differential equation and a partial differential equation is the connection between the Navier-Stokes equation and the Foiaş equation. Investigation on this connection by nonstandard methods was done in [6] and [7].

Diffusion processes $Y: \Omega \times\left[t_{0}, T\right] \mapsto \mathbb{R}$ with initial state $x_{0}$ are in the standard mathematical literature described as solutions of stochastic differential equations

$$
d Y_{t}=a\left(t, Y_{t}\right) d t+\sigma\left(t, Y_{t}\right) d B_{t} \wedge Y_{t_{0}}=x_{0}
$$

(with $B_{t}$ denoting Brownian motion). Note that the existence of the solution of a stochastic differential equation presupposes in the Ito interpretation ([28] Section 5.1) the notion of stochastic integration. Equation (1) is in the Ito interpretation just an abbreviation of $Y_{T}=Y_{t_{0}}+\int_{t_{0}}^{T} a\left(t, Y_{t}\right) d t+\int_{t_{0}}^{T} \sigma\left(t, Y_{t}\right) d B_{t}$. (See also [31] Section 3.4 or [16] Sections 5.2 and 5.3.) 
In our approach we investigate the solution $X: \Omega \times\left[t_{0} \ldots T\right] \rightarrow \mathbb{R}$ of the infinitesimal stochastic difference equation

$$
\delta X_{t}=a\left(t, X_{t}\right) d t+\sigma\left(t, X_{t}\right) \delta W_{t} \text { with initial state } X_{t_{0}}=x_{0} .
$$

Here $\left(\delta W_{t}\right)_{t \in\left[t_{0} \ldots T\right)}$ denotes an arbitrary infinitesimal model of white noise (see Definition 3.11). Note that our approach does not rely on the concept of Ito integration. We also do not use martingale arguments, Ito's formula, Girsanov's theorem, Nelson's reduction algorithm, Loeb measures, combinatorics or the Fourier-Laplace transform.

The existence of solutions $u:\left[t_{0}, T\right] \times \mathbb{R} \rightarrow \mathbb{R}$ of Kolmogorov's backward equation

$$
\frac{\partial u(t, x)}{\partial t}+a(t, x) \frac{\partial u(t, x)}{\partial x}+\frac{1}{2} \sigma^{2}(t, x) \frac{\partial^{2} u(t, x)}{\partial x^{2}}=0, \quad u(T, x)=f(x)
$$

constitutes the starting point of our investigation. Sufficient regularity conditions on the functions $a, \sigma$ and $f$ that grant the existence of a solution ${ }^{4}$ of Kolmogorov's backward equation are provided in [34] Theorem 3.2.1 and [18] Theorem 4.8.6. An analytic existence proof can be found in [13] Section 6.

Given a random variable $Z$ we denote its expectation by $\mathbb{E}[Z]$. The solution $u(t, x)$ of Kolmogorov's backward equation is (following [31] Section 5.1) linked to (1) via

$$
u\left(t_{0}, x_{0}\right)=\mathbb{E}\left[f \circ Y_{T}\right] .
$$

We prove (see Theorem 4.13), that the solution $X$ of (2) fulfills

$$
\mathbb{E}\left[f \circ X_{T}\right] \approx u\left(x_{0}, t_{0}\right) .
$$

The closeness of $\mathbb{E}\left[f \circ X_{T}\right]$ and $u\left(x_{0}, t_{0}\right)$ expressed by (5) is the starting point for all further investigations. It enables us to prove a Lindeberg type limit theorem for discrete time processes that approximate diffusion processes. It further shows together with (4) that

$$
\mathbb{E}\left[f \circ X_{T}\right] \approx \mathbb{E}\left[f \circ Y_{T}\right]
$$

(provided that the expectations $\mathbb{E}\left[f \circ X_{T}\right], \mathbb{E}\left[f \circ Y_{T}\right]$ and the solution $u$ of (3) exist).

\footnotetext{
${ }^{4}$ If $u(t, x)$ is a solution of the terminal value problem (3), then $v(t, x):=u\left(T+t_{0}-t, x\right)$ solves$$
\frac{\partial v(t, x)}{\partial t}=a(t, x) \frac{\partial v(t, x)}{\partial x}+\frac{1}{2} \sigma^{2}(t, x) \frac{\partial^{2} v(t, x)}{\partial x^{2}}, v\left(t_{0}, x\right)=f(x)
$$ 


\section{Preliminaries and Notation}

We use the notation provided by internal theories to formulate our mathematical results. The notation can be obtained form the st- $\in$-languages IST or BST (see [26], [10], [15]). We suppose that the reader is familiar with the notion of standard, internal and external formula and with the principles of transfer, idealization and standardization and some elementary consequences of these notions and principles (see [26], [10]).

We denote by st or st(.) the unary predicate standard. We denote by $\mathbb{N}$ and $\mathbb{R}$ the set of all natural numbers $\{0,1,2, \ldots\}$ and the set of all real numbers, respectively, i.e., the sets $\mathbb{N}$ and $\mathbb{R}$ contain standard as well as nonstandard elements. We use the term "set" synonymously with the term "internal set". We say that a set $S$ is countable if $S$ is either finite or possesses the cardinality of $\mathbb{N}$.

For notational convenience we also write $\operatorname{nst}(x)$ instead of $\neg \operatorname{st}(x)$, we write $\forall^{\text {st }} x \phi(x)$ instead of $\forall x(\operatorname{st}(x) \Rightarrow \phi(x))$ and $\exists^{\text {st }} x \phi(x)$ instead of $\exists x(\operatorname{st}(x) \wedge \phi(x))$. The abbreviations $\forall^{\text {nst }} x \phi(x)$ and $\exists^{\text {nst }} x \phi(x)$ are used in an analogous manner.

Notation 2.1 Let $(X,\|\|$.$) be a normed space. We say that x \in(X,\|\|$.$) is limited$ and write $\|x\|<<+\infty$ if $\exists^{s t} n \in \mathbb{N}$ such that $\|x\|<n$; otherwise we say that $x$ is unlimited. In the case that $(X,\|\|)=.(\mathbb{R},||$.$) we also write -\infty<<x<<+\infty$ instead of $\|x\|<<+\infty$. For positive unlimited $r \in(\mathbb{R},||$.$) we write r \approx \infty$. We say that $x \in(X,\|\|$.$) is infinitely small or infinitesimal if \forall^{s t} \varepsilon>0\|x\|<\varepsilon$. If $x-x^{\prime}$ is infinitely small we write $x \approx x^{\prime}$. Thus if $x$ is infinitely small we write $x \approx 0$. Note that all the concepts introduced above are external.

Notation 2.2 We further introduce a symbol $\oslash$. It is used as a replacement character for a non explicitly stated infinitesimal quantity. Let $F(\xi)$ and $G(\xi)$ denote functions of a variable $\xi$ that are possibly constant in $\xi$. We define

$$
F(\oslash) \leq G(\oslash): \Longleftrightarrow \forall o_{1} \approx 0 \exists o_{2} \approx 0 \text { such that } F\left(o_{1}\right) \leq G\left(o_{2}\right) .
$$

The symbol $\oslash$ is used in the same manner if the character $\leq$ in (6) is replaced by the character $=$. For example let $F(\xi)$ be defined by $F(\xi):=\mathbb{E}\left[\delta W_{t}\right]$ independent of $\xi$ and let $G(\xi):=\xi \cdot \delta t$. Then

$$
\mathbb{E}\left[\delta W_{t}\right]=\oslash \cdot \delta t \Longleftrightarrow \exists o_{2} \approx 0 \text { such that } \mathbb{E}\left[\delta W_{t}\right]=o_{2} \cdot \delta t .
$$

Further our definition implies that

$$
\left(\forall^{\text {st }} \varepsilon>0\right)(\oslash \leq \varepsilon)
$$


since this is just an abbreviation of $\left(\forall^{\mathrm{st}} \varepsilon\right)(\forall o \approx 0)(o \leq \varepsilon)$. We also note that

$$
F(\oslash) \leq G(\oslash) \wedge G(\oslash) \leq H(\oslash) \Rightarrow F(\oslash) \leq H(\oslash)
$$

and

$$
F(\oslash) \leq G(\oslash) \Rightarrow F(\oslash)+H(\oslash) \leq G(\oslash)+H(\oslash) .
$$

For the use of the symbol $\oslash$ as an external number (differing from our use) see [19].

The following proposition is an elementary consequence of idealization. We state it, since we are going to use it explicitly in the proofs of Lemma 4.7, Theorem 5.2 and Lemma A.2 (and thus implicitly in the proofs of Theorem 4.13, Theorem 5.4 and Example 6.6).

Proposition 2.3 Let $\phi$ be an internal formula. Then

$$
\forall^{s t} \varepsilon \in(0, \infty) \phi(\varepsilon) \Longrightarrow \exists \varepsilon \in(0, \infty) \text { such that } \varepsilon \approx 0 \wedge \phi(\varepsilon) .
$$

For convenience of the reader we state the definitions of the important concepts of S-convergence and uniform S-continuity.

Definition 2.4 A sequence is a function defined on $\mathbb{N}$. Let $(Y,\|\|$.$) be a normed space.$ We say that the sequence

$$
\left(y_{n}\right)_{n \in \mathbb{N}} \in Y^{\mathbb{N}} \text { S-converges to } y_{\infty} \in(Y,\|.\|), \quad \text { iff } \forall^{\text {nst }} h \in \mathbb{N} y_{h} \approx y_{\infty} .
$$

Let $Z$ be a subset of a normed space $(X,\|\|$.$) . We say that a function f: Z \rightarrow Y$ is uniformly S-continuous, if

$$
\forall z, z_{0} \in Z \wedge z \approx z_{0} \Rightarrow f(z) \approx f\left(z_{0}\right)
$$

(We use the term "uniformly S-continuous" since "S-continuity" has been used in different ways, i.e., compare with [29] Theorem 4.5.8 and [10] Definition 1.3.4).

Remark 2.5 A standard sequence $\left(x_{n}\right)_{n \in \mathbb{N}}$ S-converges if and only if there exists a standard $x_{\infty}$ such that $\left(x_{n}\right)_{n \in \mathbb{N}}$ converges (in the usual ZFC-based sense) to $x_{\infty}$. A standard function $f$ is uniformly $S$-continuous if and only if it is uniformly continuous in the usual sense. (Both assertions follow from the permanence principle [underspill and overspill] and transfer.)

Definition 2.6 Let $t_{0}, T \in \mathbb{R}$ be limited. A near interval $\left[t_{0} \ldots T\right]$ is a finite subset of $\left[t_{0}, T\right]$ that contains $\left\{t_{0}, T\right\}$ and further fulfills

$$
s, s^{\prime} \in\left[t_{0}, T\right] \wedge s<s^{\prime} \wedge s t(s), \operatorname{st}\left(s^{\prime}\right) \Longrightarrow \exists t \in\left[t_{0} \ldots T\right] \cap\left(s, s^{\prime}\right) .
$$

We further use the following notations for near intervals: $\left[t_{0} \ldots T\right):=\left[t_{0} \ldots T\right] \backslash\{T\}$, $\left(t_{0} \ldots T\right]:=\left[t_{0} \ldots T\right] \backslash\left\{t_{0}\right\}$ and $\left(t_{0} \ldots T\right):=\left[t_{0} \ldots T\right] \backslash\left\{t_{0}, T\right\}$. 


\section{Some probabilistic concepts}

For the purpose of simplicity we suppose that the random variables under consideration are defined on a countable set $\Omega$, endowed with a probability $\mathbb{P}$ determined point wise by its values $\mathbb{P}(\{\omega\})$, i.e., $\mathbb{P}$ is defined on the power set $\mathcal{P}(\Omega)$ of $\Omega, \mathbb{P}$ is countably additive and thus determined by its values $\mathbb{P}(\{\omega\})$ on $\{\{\omega\} \mid \omega \in \Omega\}$.

Let $(X, \mathcal{A})$ be an arbitrary measurable space. We note that any internal function $f:(\Omega, \mathcal{P}(\Omega)) \mapsto(X, \mathcal{A})$ is measurable. Thus our restriction to countable $\Omega$ relieves us from measurability arguments. However there is a close relation between internal and Loeb-measurable mappings for measure spaces [8], [21] and stochastic processes [12]).

\section{Distribution and Convergence of Random Variables}

Definition 3.1 We denote by $\mathcal{C}_{0}$ the space of all continuous functions $f: \mathbb{R} \rightarrow \mathbb{R}$ with compact support. For $n \in \mathbb{N} \cup\{\infty\}$ we denote by $\mathcal{C}^{n}$ the space of all $n$-times continuously differentiable functions $f: \mathbb{R} \rightarrow \mathbb{R}$. We further let $\mathcal{C}_{0}^{n}:=\mathcal{C}^{n} \cap \mathcal{C}_{0}$. Note that $f \in \mathcal{C}_{0}^{n}$ implies that all derivatives of $f$ up to order $n$ are uniformly continuous.

Definition 3.2 (Compare with [27] Chapter 7.) We say that a random variable $Y$ is almost limited if for any standard $\varepsilon>0$ there exists a standard $n \in \mathbb{N}$ such that $\mathbb{P}(Y \in[-n, n])>1-\varepsilon$.

Definition 3.3 Let $Y: \Omega \rightarrow \mathbb{R}$ be an almost limited random variable and let $Q$ : $\mathbb{R} \rightarrow[0,1]$ be a monotone increasing function such that $0=\lim _{x \rightarrow-\infty} Q(x)=1-$ $\lim _{x \rightarrow \infty} Q(x)$. We say that $Y$ possesses approximately a law (distribution, cumulative distribution function) $Q: \mathbb{R} \rightarrow[0,1]$, or we say that $Y$ is approximately $Q$-distributed, if

$$
\left(\forall^{\mathrm{st}} f \in \mathcal{C}_{0}\right)\left(\mathbb{E}[f \circ Y] \approx \int f(y) d Q(y)\right),
$$

where $\int f(y) d Q(y)$ denotes the Riemann-Stieltjes integral of $f$ with respect to $Q$ and $\mathbb{E}[f \circ Y]$ denotes the expectation of the random variable $f \circ Y: \Omega \rightarrow \mathbb{R}$.

Proposition 3.4 Let $Y: \Omega \rightarrow \mathbb{R}$ be an almost limited random variable. $Y$ possesses approximately a distribution $Q$ if and only if

$$
\left(\forall^{s t} g \in \mathcal{C}_{0}^{\infty}\right) \quad\left(\mathbb{E}[g \circ Y] \approx \int g(y) d Q(y)\right) .
$$


Proof: All we have to show is, that (8) implies (7). By the Stone-Weierstrass-Theorem and transfer any standard $f \in \mathcal{C}_{0}$ can be uniformly approximated up to $\frac{\varepsilon}{3}$ for an arbitrary standard $\varepsilon>0$ by a standard function $g \in \mathcal{C}_{0}^{\infty}$. Thus (8) implies

$$
\left|\mathbb{E}[f \circ Y]-\int f d Q\right| \leq\left|\mathbb{E}[g \circ Y]-\int g d Q\right|+2 \frac{\varepsilon}{3} \leq \oslash+2 \frac{\varepsilon}{3} \leq \varepsilon .
$$

Since $\varepsilon>0$ was assumed to be standard but otherwise arbitrary and $f \in \mathcal{C}_{0}$ was an arbitrary standard function, we obtain that (9) implies (7).

Definition 3.5 Let $\left(Y_{i}\right)_{i \in \mathbb{N}}$ be a sequence of almost limited random variables $Y_{i}: \Omega \rightarrow$ $\mathbb{R}$. We say that $\left(Y_{i}\right)_{i \in \mathbb{N}} S$-converges approximately to $Y_{\infty}: \Omega \rightarrow \mathbb{R}$ if

$$
\left(\forall^{\mathrm{st}} f \in \mathcal{C}_{0}\right)\left(\forall^{\mathrm{nst}} i \in \mathbb{N}\right)\left(\mathbb{E}\left[f \circ Y_{i}\right] \approx \mathbb{E}\left[f \circ Y_{\infty}\right]\right)
$$

Proposition 3.6 Let $\left(Y_{i}\right)_{i \in \mathbb{N}}$ be a sequence of almost limited random variables $Y_{i}$ : $\Omega \rightarrow \mathbb{R} .\left(Y_{i}\right)_{i \in \mathbb{N}} S$-converges approximately to a random variable $Y_{\infty}: \Omega \rightarrow \mathbb{R}$ if and only if

$$
\left(\forall^{s t} t_{\left.g \in \mathcal{C}_{0}^{\infty}\right)\left(\forall^{n s t}\right.} i \in \mathbb{N}\right)\left(\mathbb{E}\left[g \circ Y_{i}\right] \approx \mathbb{E}\left[g \circ Y_{\infty}\right]\right)
$$

The random variable $Y_{\infty}$ is again almost limited.

Proof: The proof of Proposition 3.6 is analogous to the proof of Proposition 3.4.

Proposition 3.7 Let a sequence $\left(Y_{i}\right)_{i \in \mathbb{N}}$ of almost limited random variables $Y_{i}$ and a standard sequence $\left(Q_{i}\right)_{i \in \mathbb{N}}$ of distributions $Q_{i}$ be given. Suppose that $Y_{i}$ is $Q_{i}$ distributed. Then $\left(Q_{i}\right)_{i \in \mathbb{N}}$ converges weakly to a standard distribution $Q_{\infty}: \mathbb{R} \rightarrow \mathbb{R}$, i.e.,

$$
\left(\forall f \in \mathcal{C}_{0}\right)\left(\lim _{i \rightarrow \infty} \int f d Q_{i}=\int f d Q_{\infty}\right)
$$

if and only if

$$
\left(\forall^{s t} g \in \mathcal{C}_{0}^{\infty}\right)\left(\forall^{n s t} i \in \mathbb{N}\right)\left(\mathbb{E}\left[g \circ Y_{i}\right] \approx \int g d Q_{\infty}\right)
$$

Proof: (10) holds by transfer if and only if

$$
\left(\forall^{s t} f \in \mathcal{C}_{0}\right)\left(\lim _{i \rightarrow \infty} \int f d Q_{i}=\int f d Q_{\infty}\right)
$$

thus further by Remark 2.5 if and only if

$$
\left(\forall^{s t} f \in \mathcal{C}_{0}\right)\left(\forall^{n s t} i \in \mathbb{N}\right)\left(\int f d Q_{i} \approx \int f d Q_{\infty}\right)
$$


and further by Definition 3.3 if and only if

$$
\left(\forall^{s t} f \in \mathcal{C}_{0}\right)\left(\forall^{n s t} i \in \mathbb{N}\right)\left(\mathbb{E}\left[f \circ Y_{i}\right] \approx \int f d Q_{\infty}\right)
$$

and finally by Proposition 3.4 if and only if (11) holds.

Remark 3.8 For the definition of weak convergence see [30] definition B.80. For results on weak convergence in the setting of Loeb measure spaces consult [2].

Remark 3.9 We may define (analogous to [27] Chapter 17) that two random variables $X, Y: \Omega \rightarrow \mathbb{R}$ are nearly equivalent if for all limited, S-continuous $h: \mathbb{R} \rightarrow \mathbb{R}$ we are given that $\mathbb{E}[h \circ X] \approx \mathbb{E}[h \circ Y]$. Suppose that $X: \Omega \rightarrow \mathbb{R}$ is an almost limited random variable and let $Y$ be a second random variable. By the fact that for any limited, S-continuous $h: \mathbb{R} \rightarrow \mathbb{R}$, any standard $\varepsilon>0$ and any standard $n \in \mathbb{N}$ there exists a standard $f \in \mathcal{C}_{0}$ such that

$$
\forall x \in[-n, n] \quad|f(x)-h(x)| \leq \varepsilon
$$

we obtain that $X$ and $Y$ are nearly equivalent if and only if

$$
\forall^{s t} f \in \mathcal{C}_{0} \quad \mathbb{E}[f \circ X] \approx \mathbb{E}[f \circ Y]
$$

This relates the concept of near equivalence to the concept of approximate $S$-convergence and further shows that for almost limited $X$ the random variables $X$ and $Y$ are nearly equivalent if and only if they possess a common distribution $Q$.

\section{Stochastic processes}

Notation 3.10 For convenience of notation we switch freely between the following formulations of the concept of a stochastic process $X$ with time $\left[t_{0} \ldots T\right]$ and state space $M$ :

$$
X: \Omega \times\left[t_{0} \ldots T\right] \rightarrow M, \quad X: \Omega \rightarrow M^{\left[t_{0} \ldots T\right)} \text { and } X:\left[t_{0} \ldots T\right) \rightarrow M^{\Omega} .
$$

Definition 3.11 Let $\left[t_{0} \ldots T\right]$ be a near interval and let $\left(\delta W_{t}\right)_{t \in\left[t_{0} \ldots T\right)}$ denote an indexed family of independent random variables $\delta W_{t}: \Omega \mapsto \mathbb{R}$ with laws $\nu_{t}$ such that

$$
\mathbb{E}\left[\delta W_{t}\right]=\oslash \cdot \delta t, \quad \operatorname{Var}\left(\delta W_{t}\right)=(1+\oslash) \cdot \delta t
$$

and such that

$$
(\exists \eta \approx 0) \quad\left(\sum_{t \in\left[t_{0} \ldots T\right)}\left[\int_{|y| \geq \eta} y^{2} d \nu_{t}(y)\right]<\eta\right) .
$$


Define further a stochastic process $W$ by

$$
W_{t}:=\sum_{s \in\left[t_{0} \ldots t\right)} \delta W_{s} .
$$

We say that $\left(\delta W_{t}\right)_{t \in\left[t_{0} \ldots T\right)}$ is a model of white noise and that $W$ is a model of Brownian motion.

Remark 3.12 Note that (12) is an infinitesimal formulation of the Lindeberg condition. It is equivalent with the near Lindeberg condition (14.1) in [27]. A precise statement of the equivalence together with a proof is provided in the appendix of this article.

Definition 3.13 Let $\left[t_{0} \ldots T\right]$ be a near interval and let $\left(\delta W_{t}\right)_{t \in\left[t_{0} \ldots T\right)}$ be a model of white noise. Let functions

$$
a:\left[t_{0}, T\right] \times \mathbb{R} \rightarrow \mathbb{R} \text { and } \sigma:\left[t_{0}, T\right] \times \mathbb{R} \rightarrow \mathbb{R}
$$

be given. Let

$$
[K(t, x)](\omega):=x+a(t, x) \delta t+\sigma(t, x) \delta W_{t}(\omega) .
$$

Let $x_{0} \in \mathbb{R}$ be fixed. Let a stochastic process $X: \Omega \times\left[t_{0} \ldots T\right] \rightarrow \mathbb{R}$ be recursively defined by

$$
X_{t_{0}}(\omega):=x_{0} \text { and } X_{t+\delta t}(\omega):=\left[K\left(t, X_{t}(\omega)\right)\right](\omega) .
$$

We let $\delta X_{t}:=X_{t+\delta t}-X_{t}$ and say that the process $X$ is the solution of the infinitesimal stochastic difference equation

$$
\delta X_{t}=a\left(t, X_{t}\right) \delta t+\sigma\left(t, X_{t}\right) \delta W_{t} .
$$

with initial condition $X_{t_{0}}=x_{0}$. 


\section{Diffusion Equations and Processes}

Definition 4.1 Given a function $u:\left[t_{0}, T\right] \times \mathbb{R} \rightarrow \mathbb{R}$, we say that $u$ is $\mathcal{C}_{b}^{m, n}$ if $u$ is a bounded uniformly continuous function such that all its derivatives up to order $m$ with respect to its first variable and all its derivatives up to order $n$ with respect to its second variable are bounded and uniformly continuous (as functions of both variables).

Provided that the respective derivatives exist, we introduce the following notations:

Notation 4.2 Let $\psi: \mathbb{R} \rightarrow \mathbb{R}$. We denote by $\psi^{\prime}(x)$ the first order derivative of $\psi$ at $x$ and by $\psi^{\prime \prime}(x)$ the second order derivative of $\psi$ at $x$. Given a function $u:\left[t_{0}, T\right] \times \mathbb{R} \rightarrow$ $\mathbb{R}$, we denote the partial derivative of $u$ with respect to its first variable at $(t, x)$ by

$$
\left.\frac{\partial u(s, x)}{\partial s}\right|_{s=t} \text { resp. for simplicity of notation by } \frac{\partial u(t, x)}{\partial t} .
$$

We denote the first and second order derivatives of $u$ with respect to its second variable by

$$
\left.\frac{\partial u(t, y)}{\partial y}\right|_{y=x} \text { and }\left.\frac{\partial^{2} u(t, y)}{\partial^{2} y}\right|_{y=x} .
$$

resp. for simplicity of notation by

$$
\frac{\partial u(t, x)}{\partial x} \text { and } \frac{\partial^{2} u(t, x)}{\partial^{2} x}
$$

Proposition 4.3 Let $x \in \mathbb{R}$ and let $v: \mathbb{R} \rightarrow \mathbb{R}$ be a function such that $v(x)=0$ and $v^{\prime}(x)=0$. Suppose further that $x \mapsto v^{\prime \prime}(x)$ is $S$-continuous and limited. Then

$$
y \approx 0 \Longrightarrow v(x+y)=\frac{1}{2}\left(v^{\prime \prime}(x)+\oslash\right) \cdot y^{2} .
$$

Definition 4.4 Given a one times differentiable function $v: \mathbb{R} \rightarrow \mathbb{R}$, we let

$$
\left[R_{x} v\right](x+y):=v(x+y)-v(x)-v^{\prime}(x) \cdot y .
$$

and call $R_{x} v$ the remainder of $v$ at $x$.

Definition 4.5 We denote by $\left(\mathcal{L}_{t}: \mathcal{C}^{2} \rightarrow \mathcal{C}^{0}\right)_{t \in\left[t_{0}, T\right]}$ a standard indexed family of differential operators of the form

$$
\left[\mathcal{L}_{t} \psi\right](x):=\frac{1}{2} \psi^{\prime \prime}(x) \cdot b(t, x)+\psi^{\prime}(x) \cdot a(t, x),
$$


with $a:\left[t_{0}, T\right] \times \mathbb{R} \rightarrow \mathbb{R}$ and $b:\left[t_{0}, T\right] \times \mathbb{R} \rightarrow[0, \infty)$ bounded functions. Let

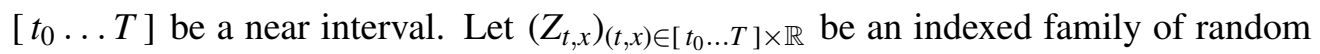
variables

$$
Z_{t, x}: \Omega \rightarrow \mathbb{R} \text { with laws } \nu_{t, x}
$$

We say that $\left(Z_{t, x}\right)_{(t, x) \in\left[t_{0} \ldots T\right] \times \mathbb{R}}$ is associated with the family of operators $\left(\mathcal{L}_{t}\right)_{t \in\left[t_{0}, T\right]}$ if:

(i) $\mathbb{E}\left[Z_{t, x}\right]=(a(t, x)+\oslash) \cdot \delta t$

(ii) $\operatorname{Var}\left(Z_{t, x}\right)=(b(t, x)+\oslash) \cdot \delta t$.

There exists an infinitesimal $\eta>0$ such that

(iii) $\sum_{t \in\left[t_{0} \ldots T\right)} \sup _{x \in \mathbb{R}}\left[\int_{|y| \geq \eta} y^{2} d \nu_{t, x}(y)\right]<\eta$.

\section{Remark 4.6 Let}

$$
\delta \eta_{t}:=\sup _{x \in \mathbb{R}}\left[\int_{|y| \geq \eta} y^{2} d \nu_{t, x}(y)\right] \geq 0 .
$$

Then (iii) of Definition 4.5 becomes

$$
\sum_{t \in\left[t_{0} \ldots T\right)} \delta \eta_{t}<\eta \approx 0
$$

Let $\widetilde{Z}_{t, x}$ be the random variable defined by

$$
\widetilde{Z}_{t, x}(\omega):=\left\{\begin{array}{l}
Z_{t, x}(\omega) \text { if }\left|Z_{t, x}(\omega)\right|<\eta \text { and } \\
0 \text { else. }
\end{array}\right.
$$

Then by (13) and (15)

$$
\mathbb{E}\left[\widetilde{Z}_{t, x}^{2}\right]=\mathbb{E}\left[Z_{t, x}^{2}\right]+O_{t, x} \text { with }\left|O_{t, x}\right| \leq \delta \eta_{t} .
$$

Further

$$
\begin{aligned}
\mathbb{E}\left[Z_{t, x}^{2}\right] & =\operatorname{Var}\left(Z_{t, x}\right)+\mathbb{E}\left[Z_{t, x}\right]^{2} \\
& =(b(t, x)+\oslash) \cdot \delta t+(a(t, x)+\oslash)^{2} \cdot(\delta t)^{2}
\end{aligned}
$$


Lemma 4.7 Let $\left(\mathcal{L}_{t}\right)_{t \in\left[t_{0}, T\right]}$ be a standard indexed family of differential operators that fulfills the hypotheses of Definition 4.5. Let $f: \mathbb{R} \rightarrow \mathbb{R}$ be a standard bounded $\mathcal{C}^{2}$ function. Suppose that there exists a $\mathcal{C}_{b}^{1,2}$ solution $u:\left[t_{0}, T\right] \times \mathbb{R} \rightarrow \mathbb{R}$ of the terminal value problem

$$
\left[\mathcal{L}_{t} u(t, .)\right](x)+\frac{\partial u(t, x)}{\partial t}=0, \quad u(T, x)=f(x) .
$$

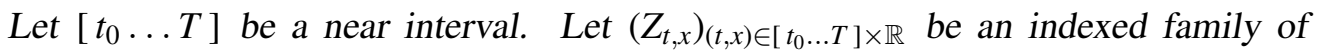
random variables that is associated with the family of operators $\left(\mathcal{L}_{t}\right)_{t \in\left[t_{0}, T\right]}$. Let $\hat{u}:\left[t_{0} \ldots T\right] \times \mathbb{R} \rightarrow \mathbb{R}$ be defined by backward induction on $\left[t_{0} \ldots T\right]$ by

$$
\hat{u}(T, x):=f(x) \text { and } \hat{u}(t, x):=\mathbb{E}\left[\hat{u}\left(t+\delta t, x+Z_{t, x}\right)\right] .
$$

Then

$$
\text { for any }(s, x) \in\left[t_{0} \ldots T\right] \times \mathbb{R} \text { we have } \hat{u}(s, x) \approx u(s, x) \text {. }
$$

Remark 4.8 Note that by standardness of $\left(\mathcal{L}_{t}: \mathcal{C}^{2} \rightarrow \mathcal{C}^{0}\right)_{t \in\left[t_{0}, T\right]}$ it follows that $b(.,$. and $a(.,$.$) are standard functions and that \left[t_{0}, T\right]$ is a standard interval.

Corollary 4.9 The solution $u(.,$.$) of (18) presumed in Lemma 4.7$ is standard and unique.

Proof: By standardness of $\left(\mathcal{L}_{t}\right)_{t \in\left[t_{0}, T\right]}$ and $f$, and by the existence of a continuous solution $u$ of (18), we obtain by transfer that there exists a continuous standard solution $u$ of (18). For a given family $\left(Z_{t, x}\right)_{(t, x) \in\left[t_{0} \ldots T\right)}$ of random variables $Z_{t, x}: \Omega \rightarrow \mathbb{R}$ associated with $\left(\mathcal{L}_{t}\right)_{t \in\left[t_{0} \ldots T\right]}$ the function $\hat{u}$ is well defined. Thus there exists at most one continuous standard function $u:\left[t_{0} \ldots T\right] \times \mathbb{R} \rightarrow \mathbb{R}$ such that (20) holds.

Remark 4.10 Note that uniqueness results for solutions of diffusion equations (compare with [33] sections 11.3 and 11.5) are non trivial.

Notation 4.11 For simplicity of notation we write $u_{t}(x)$ instead of $u(t, x)$ if this seems convenient to us.

Remark 4.12 The proof of Lemma 4.7 following this remark consists of four parts. In part I we collect facts concerning the solution $u$ of (18) that are used in part II. Letting $\gamma_{t, x}:=\mathbb{E}\left[u\left(t+\delta t, x+Z_{t, x}\right)\right]-u(t+\delta t, x)$ we obtain in part II a bound for $\left|\gamma_{t, x}-\delta t \cdot\left[\mathcal{L}_{t} u(t+\delta t,).\right](x)\right|$ (compare with (28)). This result of part II is used in part III to obtain a bound for $\left|\mathbb{E}\left[u\left(t+\delta t, x+Z_{t, x}\right)\right]-u(t, x)\right|$ (compare with (32)). From the bound in part III we obtain finally a bound of $|\hat{u}(s, x)-u(s, x)|$ (compare with (36)) in part IV. 
Proof of Lemma 4.7:

Part I: Note that by Definition 4.4

$$
\left[R_{x} u_{t+\delta t}\right](x+y)=u_{t+\delta t}(x+y)-u_{t+\delta t}(x)-u_{t+\delta t}^{\prime}(x) \cdot y .
$$

We further remark that

$$
\begin{gathered}
{\left[R_{x} u_{t+\delta t}\right]^{\prime \prime}(x+y)=u_{t+\delta t}^{\prime \prime}(x+y),} \\
{\left[R_{x} u_{t+\delta t}\right](x)=0 \text { and }\left[R_{x} u_{t+\delta t}\right]^{\prime}(x)=0 .}
\end{gathered}
$$

By the hypothesis that $u \in \mathcal{C}_{b}^{1,2}$ and the fact that $u(.,$.$) is standard by Corollary 4.9,$ there exists a standard $\rho \in \mathbb{R}$ such that

$$
\forall(t, x) \in\left[t_{0}, T\right] \times \mathbb{R} \quad\left|u_{t+\delta t}(x)\right|,\left|u_{t+\delta t}^{\prime}(x)\right|,\left|u_{t+\delta t}^{\prime \prime}(x)\right|<\rho
$$

and for any $t \in\left[t_{0}, T\right]$ we have that

$$
x \mapsto u_{t}^{\prime \prime}(x) \text { is uniformly S-continuous. }
$$

Note that by (22), (23) and (24) we obtain

$$
\left[R_{x} u_{t+\delta t}\right](x+y) \leq \frac{1}{2} \rho y^{2} .
$$

Let

$$
\gamma_{t, x}:=\mathbb{E}\left[u\left(t+\delta t, x+Z_{t, x}\right)\right]-u(t+\delta t, x) .
$$

Part II: We show that for $x \in \mathbb{R}$ and $t \in\left[t_{0} \ldots T\right.$ )

$$
\left|\gamma_{t, x}-\delta t \cdot\left[\mathcal{L}_{t} u(t+\delta t, .)\right](x)\right| \leq \delta \eta_{t} \cdot c+\delta t \cdot \oslash
$$

with

$$
c:=\frac{1}{\sqrt{\eta}} \approx \infty .
$$

To do this we have to show that for $x \in \mathbb{R}$ and $t \in\left[t_{0} \ldots T\right)$

$$
\gamma_{t, x} \leq \delta \eta_{t} \cdot c+\delta t \cdot \oslash+\delta t\left[\mathcal{L}_{t} u(t+\delta t, .)\right](x)
$$

and

$$
\gamma_{t, x} \geq-\left(\delta \eta_{t} \cdot c+\delta t \cdot \oslash\right)+\delta t\left[\mathcal{L}_{t} u(t+\delta t, .)\right](x) .
$$

Since the proofs of (30) and (31) are completely analogous, we just prove (30) and regard (31) and thus further (28) as being proved. 
Let now $x \in \mathbb{R}$ and $t \in\left[t_{0} \ldots T\right)$ be arbitrarily given. Then

$$
\begin{aligned}
& \gamma_{t, x}=\mathbb{E}\left[u\left(t+\delta t, x+Z_{t, x}\right)\right]-u(t+\delta t, x) \\
& \stackrel{(a)}{=} \mathbb{E}\left[\left[R_{x} u_{t+\delta t}\right]\left(x+Z_{t, x}\right)\right]+\mathbb{E}\left[u_{t+\delta t}^{\prime}(x) \cdot Z_{t, x}\right] \\
& \stackrel{(b)}{=} \int_{|y| \geq \eta}\left[R_{x} u_{t+\delta t}\right](x+y) d \nu_{t, x}(y) \int_{|y|<\eta}\left[R_{x} u_{t+\delta t}\right](x+y) d \nu_{t, x}(y) \\
& +u_{t+\delta t}^{\prime}(x) \cdot(a(t, x)+\oslash) \cdot \delta t \\
& \stackrel{(c)}{\leq} \frac{1}{2} \rho \cdot \sup _{x \in \mathbb{R}} \int_{|y| \geq \eta}|y|^{2} d \nu_{t, x}(y)+\int_{|y|<\eta} \frac{1}{2} \cdot\left[u_{t+\delta t}^{\prime \prime}(x)+\oslash\right] \cdot y^{2} d \nu_{t, x}(y) \\
& +\delta t \cdot u_{t+\delta t}^{\prime}(x) \cdot(a(t, x)+\oslash) \\
& \stackrel{(d)}{=} \frac{1}{2} \rho \cdot \delta \eta_{t}+\frac{1}{2} \cdot\left[u_{t+\delta t}^{\prime \prime}(x)+\oslash\right] \cdot \mathbb{E}\left[\tilde{Z}_{t, x}^{2}\right]+\delta t \cdot u_{t+\delta t}^{\prime}(x) \cdot(a(t, x)+\oslash) \\
& \stackrel{(e)}{=} \delta \eta_{t} \cdot \frac{1}{2} \rho+\frac{1}{2}\left[u_{t+\delta t}^{\prime \prime}(x)+\oslash\right] \cdot\left(\mathbb{E}\left[Z_{t, x}^{2}\right]+O_{t, x}\right)+\delta t \cdot u_{t+\delta t}^{\prime}(x) \cdot(a(t, x)+\oslash) \\
& \stackrel{(f)}{\leq} \delta \eta_{t} \cdot \frac{1}{2} \rho+\frac{1}{2}\left[u_{t+\delta t}^{\prime \prime}(x)+\oslash\right] \cdot O_{t, x}+\frac{1}{2}\left[u_{t+\delta t}^{\prime \prime}(x)+\oslash\right] \cdot[(b(t, x)+\oslash) \cdot \delta t] \\
& +\frac{1}{2}\left[u_{t+\delta t}^{\prime \prime}(x)+\oslash\right] \cdot\left[(a(t, x)+\oslash)^{2} \cdot(\delta t)^{2}\right]+\delta t \cdot u_{t+\delta t}^{\prime}(x) \cdot(a(t, x)+\oslash) \\
& \stackrel{(g)}{\leq} \delta \eta_{t} \cdot \frac{1}{2} \rho+\delta \eta_{t} \cdot \frac{1}{2}[\rho+1]+\delta t \cdot \frac{1}{2}\left[u_{t+\delta t}^{\prime \prime}(x)+\oslash\right] \cdot(b(t, x)+\oslash) \\
& +\delta t \cdot \oslash+\delta t \cdot u_{t+\delta t}^{\prime}(x) \cdot(a(t, x)+\oslash) \\
& \stackrel{(h)}{\leq} \delta \eta_{t} \cdot[\rho+1]+\delta t \cdot \oslash+\delta t \cdot \frac{1}{2} u_{t+\delta t}^{\prime \prime}(x) \cdot b(t, x)+\delta t \cdot u_{t+\delta t}^{\prime}(x) \cdot(a(t, x)) \\
& \stackrel{(i)}{\leq} \delta \eta_{t} \cdot c+\delta t \cdot \oslash+\delta t \cdot\left[\mathcal{L}_{t} u(t+\delta t, .)\right](x) .
\end{aligned}
$$

The equality (a) follows from an application of (21), while (b) is obtained by splitting $\mathbb{E}\left[\left[R_{x} u_{t+\delta t}\right]\left(x+Z_{t, x}\right)\right]$ into two integrals and by an application of Definition 4.5 (i). The inequality (c) follows from an application of (26), and by (22), (23), Proposition 4.3 and (25). The equality (d) follows by an application of (13) and (15) while equality (e) is obtained by (16). We further obtain (f) by the use of (17) and (g) by application of (16) and (24). The relation (h) is obtained by calculation and rearrangement of terms, 
while (i) follows from the fact that $c \approx \infty$ and the definition of $\mathcal{L}$.

Thus (30) and thus further (28) has been proved.

Part III: We show that

$$
(\forall x \in \mathbb{R})\left(\left|\mathbb{E}\left[u\left(t+\delta t, x+Z_{t, x}\right)\right]-u(t, x)\right| \leq \delta \eta_{t} \cdot c+\delta t \cdot \oslash\right) .
$$

Since we assumed that $u \in \mathcal{C}_{b}^{1,2}$ we have that $t \mapsto u_{t}^{\prime}(x), t \mapsto u_{t}^{\prime \prime}(x)$ are uniformly S-continuous mappings and thus we obtain that

$$
\left|\left[\mathcal{L}_{t} u(t+\delta t, .)\right](x)-\left[\mathcal{L}_{t} u(t, .)\right](x)\right|=\left|\left[\mathcal{L}_{t}(u(t+\delta t, .)-u(t, .))\right](x)\right| \leq \oslash
$$

From (28) and (33) we obtain for $x \in \mathbb{R}$ and $t \in\left[t_{0} \ldots T\right.$ ) that

$$
\left|\gamma_{t, x}-\delta t \cdot\left[\mathcal{L}_{t} u(t, .)\right](x)\right| \leq \delta \eta_{t} \cdot c+\delta t \cdot \oslash
$$

From (18) and

$$
u(t+\delta t, x)-u(t, x)=\delta t \cdot \frac{\partial u(t, x)}{\partial t}+\delta t \cdot \oslash
$$

we obtain for $x \in \mathbb{R}$ and $t \in\left[t_{0} \ldots T\right)$

$$
u(t+\delta t, x)-u(t, x)=-\delta t \cdot\left[\mathcal{L}_{t} u(t, .)\right](x)+\delta t \cdot \oslash .
$$

From (27), (34) and (35) we obtain for $x \in \mathbb{R}$ and $t \in\left[t_{0} \ldots T\right.$ ) that

$$
\begin{aligned}
\mid \mathbb{E}\left[u\left(t+\delta t, x+Z_{t, x}\right)\right] & -u(t, x) \mid \\
& =\left|\mathbb{E}\left[u\left(t+\delta t, x+Z_{t, x}\right)\right]-u(t+\delta t, x)+u(t+\delta t, x)-u(t, x)\right| \\
& \left.=\mid \gamma_{t, x}-\delta t \cdot\left[\mathcal{L}_{t} u(t, .)\right](x)\right]+\delta t \cdot \oslash \mid \leq \delta \eta_{t} \cdot c+\delta t \cdot \oslash .
\end{aligned}
$$

i.e., (32) has been proved.

Part IV: We prove now by backward induction on $\left[t_{0} \ldots T\right]$ that for $s \in\left[t_{0} \ldots T\right]$

$$
(\forall x \in \mathbb{R}) \quad\left(|\hat{u}(s, x)-u(s, x)| \leq \sum_{r \in[s \ldots T)}\left[\delta \eta_{r} \cdot c+\delta r \cdot \oslash\right]\right) .
$$

Since (36) is external we can not apply (internal) induction directly to (36). We therefore replace (36) by an external family of formulas as follows: For any standard $\varepsilon>0$ we consider

$$
(\forall x \in \mathbb{R}) \quad\left(|\hat{u}(s, x)-u(s, x)| \leq \sum_{r \in[s \ldots T)}\left[\delta \eta_{r} \cdot c+\delta r \cdot \varepsilon\right]\right) .
$$


We prove $(\varepsilon)$ by backward induction as follows:

In the case that $s=T$ formula $(\varepsilon)$ reduces by (18) and (19) to the tautology $0 \leq 0$. To complete the proof of $(\varepsilon)$ by backward induction we just have to show that if $(\varepsilon)$ holds true for $s=t+\delta t$, then $(\varepsilon)$ holds true for $s=t$. Thus suppose that $(\varepsilon)$ holds for $s=t+\delta t$. From (19), (32) and the validity of $(\varepsilon)$ in the case $s=t+\delta t$ we obtain that for $x \in \mathbb{R}$

$$
\begin{aligned}
|\hat{u}(t, x)-u(t, x)| & =\left|\mathbb{E}\left[\hat{u}\left(t+\delta t, x+Z_{t, x}\right)\right]-u(t, x)\right| \\
& \leq\left|\mathbb{E}\left[\hat{u}\left(t+\delta t, x+Z_{t, x}\right)\right]-\mathbb{E}\left[u\left(t+\delta t, x+Z_{t, x}\right)\right]\right| \\
& +\left|\mathbb{E}\left[u\left(t+\delta t, x+Z_{t, x}\right)\right]-u(t, x)\right| \\
& \leq \sup _{z \in \mathbb{R}}|\hat{u}(t+\delta t, z)-u(t+\delta t, z)|+\left|\mathbb{E}\left[u\left(t+\delta t, x+Z_{t, x}\right)\right]-u(t, x)\right| \\
& \leq \sum_{r \in[t+\delta t \ldots T)}\left[\delta \eta_{r} \cdot c+\delta r \cdot \varepsilon\right]+\delta \eta_{t} \cdot c+\delta t \cdot \oslash \\
& \leq \sum_{r \in[t \ldots T)}\left[\delta \eta_{r} \cdot c+\delta r \cdot \varepsilon\right],
\end{aligned}
$$

i.e.,

$$
|\hat{u}(t, x)-u(t, x)| \leq \sum_{r \in[t \ldots T)}\left[\delta \eta_{r} \cdot c+\delta r \cdot \varepsilon\right]
$$

holds. Thus application of (internal) induction to (37) shows that $(\varepsilon)$ holds for any $s \in\left[t_{0} \ldots T\right]$. Since the $\varepsilon$ in our consideration was assumed to be standard but otherwise arbitrary, we obtain from Proposition 2.3 that (36) holds for all $s \in\left[t_{0} \ldots T\right]$. (36) implies by (14) and (29) that (20) holds.

Theorem 4.13 Let $\left(\mathcal{L}_{t}\right)_{t \in\left[t_{0}, T\right]}$ be a standard indexed family of differential operators that fulfills the hypotheses of Definition 4.5. Let $f: \mathbb{R} \rightarrow \mathbb{R}$ be a standard bounded $\mathcal{C}^{2}$ function. Suppose that there exists a $\mathcal{C}_{b}^{1,2}$ solution $u:\left[t_{0}, T\right] \times \mathbb{R} \rightarrow \mathbb{R}$ of the terminal value problem

$$
\left[\mathcal{L}_{t} u(t, .)\right](x)+\frac{\partial u(t, x)}{\partial t}=0, \quad u(T, x)=f(x) .
$$

Let $\left[t_{0} \ldots T\right]$ be a near interval and let $\left(\delta W_{t}\right)_{t \in\left[t_{0} \ldots T\right)}$ be a model of white noise. Let

$$
\sigma:\left[t_{0}, T\right) \times \mathbb{R} \rightarrow \mathbb{R} \text { be such that } \sigma^{2}(., .) \approx b(., .)
$$


and let $\tilde{a} \approx a$. Let $X$ be the solution of the infinitesimal stochastic difference equation

$$
\delta X_{t}=\tilde{a}\left(t, X_{t}\right) \delta t+\sigma\left(t, X_{t}\right) \delta W_{t} .
$$

with initial condition $X_{t_{0}}=x_{0}$. Then

$$
\mathbb{E}\left[f \circ X_{T}\right] \approx u\left(x_{0}, t_{0}\right) .
$$

Proof: Let us denote the conditional expectation of a random variable $Y$ with respect to a second random variable $Z$ by $\mathbb{E}[Y \mid Z]$. We apply Lemma 4.7 with

$$
Z_{t, x}:=\tilde{a}(t, x) \delta t+\sigma(t, x) \delta W_{t} .
$$

From the definition of $\hat{u}$ by (19) we obtain that

$$
\begin{array}{r}
\mathbb{E}\left[\hat{u}\left(t+\delta t, X_{t+\delta t}\right)\right]=\mathbb{E}\left[\mathbb{E}\left[\hat{u}\left(t+\delta t, X_{t+\delta t}\right) \mid X_{t}\right]\right]= \\
\mathbb{E}\left[\mathbb{E}\left[\hat{u}\left(t+\delta t, X_{t}+Z_{t, X_{t}}\right) \mid X_{t}\right]=\mathbb{E}\left[\hat{u}\left(t, X_{t}\right)\right] .\right.
\end{array}
$$

Induction over (39) and $u(T, x)=f(x)$ gives

$$
\mathbb{E}\left[f \circ X_{T}\right]=\hat{u}\left(x_{0}, t_{0}\right) .
$$

Formula (40) gives together with the conclusion of Lemma 4.7 the approximate identity (38).

Remark 4.14 Note that boundedness (and standardness) have been the only hypotheses imposed on the functions $a$ and $b$. Our proofs worked out mainly because of the strong hypotheses that the solution $u$ of (18) is a (standard) element of $\mathcal{C}_{b}^{1,2}$.

Remark 4.15 Since $a, b, t_{0}$ and $T$ are (by boundedness and standardness) limited, we obtain that the random variables $X_{t}$ - that constitute the stochastic processes under consideration - fulfill $-\infty<<\mathbb{E}\left[X_{t}\right], \operatorname{Var}\left(X_{t}\right)<<\infty$. From this we obtain further by [5] Theorem 2.11 that the random variables $X_{T}$ are almost limited. 


\section{Lindeberg's Theorem and related results}

We consider now equation (18) in the case that $b(t, x)=1, a(t, x)=0$ and $f \in \mathcal{C}_{0}^{2}$, i.e., we consider the following special case of the terminal value problem (18):

$$
\frac{1}{2} \cdot \frac{\partial^{2} u(t, x)}{\partial x^{2}}+\frac{\partial u(t, x)}{\partial t}=0, \quad u(T, x)=f(x) .
$$

A solution of the terminal value problem $(41)$ on $\left[t_{0}, T\right] \times \mathbb{R}$ is provided by the function $u$ given by

$$
u(t, x)=\int_{\mathbb{R}} f(y) \frac{1}{\sqrt{2 \pi(T-t)}} e^{-\frac{(y-x)^{2}}{2(T-t)}} d y
$$

for $(t, x) \in\left[t_{0}, T\right) \times \mathbb{R}$ and $u(T, x)=f(x)$. If $\phi$ denotes the density function of the $N(0,1)$ distribution, then also

$$
u(t, x)=\int_{y \in \mathbb{R}} \frac{1}{\sqrt{T-t}} \phi\left(\frac{y-x}{\sqrt{T-t}}\right) f(y) d y .
$$

Definition 5.1 Let $\Xi=\left(\xi_{i, j}\right)_{(i, j) \in \mathbb{N} \times \mathbb{N}}$ be an array of random variables $\xi_{i, j} \in \mathbb{R}$. Suppose that there exists a function $i \mapsto J_{i}$ from $\mathbb{N}$ to $\mathbb{N}$ such that $\lim _{i \rightarrow \infty} J_{i}=\infty$ and that $\operatorname{Var}\left(\xi_{i, j}\right)=0 \equiv \xi_{i, j}$ if and only if $j \geq J_{i}$. Suppose further that for any fixed $i$ the vector $\left.\left(\xi_{i, j}\right)\right)_{j \in \mathbb{N}}$ consists of independent random variables. We call $\Xi$ a triangular array of independent random variables with sum-variables $\zeta_{i}:=\sum_{0 \leq j \leq J_{i}-1} \xi_{i, j}=\sum_{j \in \mathbb{N}} \xi_{i, j}$.

The following theorem is known as Lindeberg's limit theorem. (Compare with [11] Section 9.6.)

Theorem 5.2 Let $\Xi$ be a triangular array of independent random variables with sumvariables $\zeta_{i}$. Suppose that $\mathbb{E}\left[\xi_{i, j}\right]=0$ for all $i, j \in \mathbb{N}$. Suppose further that

$$
\lim _{i \rightarrow \infty} \operatorname{Var}\left(\zeta_{i}\right)=\sigma^{2}
$$

and that

$$
(\forall \varepsilon>0)(\exists N \in \mathbb{N})(\forall i>N)\left(\sum_{j=0}^{J_{i}-1} \mathbb{E}\left[\xi_{i, j}^{2} \cdot \mathbb{I}_{\left\{\left|\xi_{i, j}\right| \geq \varepsilon\right\}}\right]<\varepsilon\right) .
$$

Then the sequence of distributions of $\left(\zeta_{i}\right)_{i \in \mathbb{N}}$ converges weakly to $N\left(0, \sigma^{2}\right)$. 
Proof: The theorem stated above is a theorem in the terms of standard mathematics. We can therefore by transfer suppose that the theorem is stated with the addition that any object named in the theorem is standard. Especially we can replace (44) by

$$
\left(\forall^{s t} \varepsilon>0\right)\left(\exists^{s t} N \in \mathbb{N}\right)(\forall i>N)\left(\sum_{j=0}^{J_{i}-1} \mathbb{E}\left[\xi_{i, j}^{2} \cdot \mathbb{I}_{\left\{\left|\xi_{i, j}\right| \geq \varepsilon\right\}}\right]<\varepsilon\right)
$$

and assume that $\left(\zeta_{i}\right)_{i \in \mathbb{N}}$ is a standard sequence. Assertion (45) implies that

$$
\left(\forall^{\mathrm{nst}} i \in \mathbb{N}\right)\left(\forall^{s t} \varepsilon>0\right)\left(\sum_{j=0}^{J_{i}-1} \mathbb{E}\left[\xi_{i, j}^{2} \cdot \mathbb{I}_{\left\{\left|\xi_{i, j}\right| \geq \varepsilon\right\}}\right]<\varepsilon\right) .
$$

From (46) we obtain by Proposition 2.3 that

$$
\left(\forall^{\mathrm{nst}} i \in \mathbb{N}\right)(\exists \varepsilon \approx 0)\left(\sum_{j=0}^{J_{i}-1} \mathbb{E}\left[\xi_{i, j}^{2} \cdot \mathbb{I}_{\left\{\left|\xi_{i, j}\right| \geq \varepsilon\right\}}\right]<\varepsilon\right) .
$$

From (43) we obtain by standardness of $\left(\zeta_{i}\right)_{i \in \mathbb{N}}$ and Remark 2.5 that

$$
\left(\forall^{\mathrm{nst}} i \in \mathbb{N}\right)\left(\operatorname{Var}\left(\zeta_{i}\right)=\sigma^{2}+\oslash\right) .
$$

Choose an arbitrary nonstandard $i \in \mathbb{N}$. Let for $j \in\left\{0, \ldots, J_{i}-1\right\}$ increments $\delta t_{j}$ be given, such that

$$
\delta t_{j}:=\operatorname{Var}\left(\xi_{i, j}\right) \cdot(1+\oslash) \text { and } \sum_{j=0}^{J_{i}-1} \delta t_{j}=\sigma^{2}
$$

and define points $t_{j}$ by

$$
t_{0}:=0, t_{j+1}:=t_{j}+\delta t_{j}, T:=t_{J_{i}}=\sigma^{2}
$$

Let

$$
\left[t_{0} \ldots T\right]=\left\{t_{j} \mid 0 \leq j \leq J_{i}\right\}
$$

Let $\delta W_{t_{j}}=\xi_{i, j}$ for $0 \leq j \leq J_{i}$. Then $\left(\delta W_{t}\right)_{t \in\left[t_{0} \ldots, T\right)}$ is a model of white noise. Let $W$ be the model of Brownian motion associated with $\left(\delta W_{t}\right)_{t \in\left[t_{0} \ldots T\right)}$. Then

$$
W_{T}=\zeta_{i}
$$

and $W$ is the solution $X$ of the infinitesimal stochastic difference equation $\delta X_{t}=$ $a(t, x) \delta t+b(t, x) \delta W_{t}$ with $a(t, x)=0$ and $b(t, x)=1$. Thus by Theorem 4.13 we have that for any standard $f \in \mathcal{C}_{0}^{2}$

$$
\mathbb{E}\left[f \circ W_{T}\right] \approx u(0,0)
$$


with $u$ the solution (42) of the terminal value problem (41). Thus we obtain from (48), (49), (50) and (42) that

$$
\mathbb{E}\left[f \circ \zeta_{i}\right]=\mathbb{E}\left[f \circ W_{T}\right] \approx u(0,0)=\int_{\mathbb{R}} f(y) \frac{1}{\sqrt{2 \pi} \sigma} e^{-\frac{y^{2}}{2 \sigma^{2}}} d y .
$$

By (51), the fact that $i \in \mathbb{N}$ was supposed to be nonstandard but otherwise arbitrarily chosen and by Proposition 3.7 the standard sequence of distributions of the (by Remark 4.15) almost limited random variables $\left(\zeta_{i}\right)_{i \in \mathbb{N}}$ converges weakly to $N\left(0, \sigma^{2}\right)$.

Theorem 4.13 provides together with the existence of solutions of Kolmogorov's backward equation a general limit theorem for diffusions. Since our result (Theorem 5.4 stated below) includes a Lindeberg condition, we call it a Lindeberg type limit theorem.

Definition 5.3 Let $\Xi=\left(\xi_{i, j}\right)_{(i, j) \in \mathbb{N} \times \mathbb{N}}$ be an infinite triangular array of independent random variables, with sum variables $\zeta_{i}$. Let $i \mapsto J_{i}$ denote the function involved in the definition of a triangular array (Definition 5.1). We say that $\Xi$ is a triangular approximation of white noise on $\left[t_{0}, T\right]$ if:

$$
\begin{gathered}
\forall i, j \in \mathbb{N} \quad \mathbb{E}\left[\xi_{i, j}\right]=0, \\
\forall i \in \mathbb{N} \quad \operatorname{Var}\left(\zeta_{i}\right)=\left(T-t_{0}\right)
\end{gathered}
$$

and

$$
(\forall \varepsilon>0)(\exists N \in \mathbb{N})(\forall i>N)\left(\sum_{j=0}^{J_{i}-1} \mathbb{E}\left[\xi_{i, j}^{2} \cdot \mathbb{I}_{\left\{\left|\xi_{i, j}\right| \geq \varepsilon\right\}}\right]<\varepsilon\right) .
$$

Theorem 5.4 Let $\Xi$ be a triangular approximation of white noise on $\left[t_{0}, T\right]$. Let $\left(\mathcal{L}_{t}\right)_{t \in\left[t_{0}, T\right]}$ be a family of differential operators that fulfill - with the exception of standardness - all hypotheses of Definition 4.5. Let

$$
\sigma:\left[t_{0}, T\right] \times \mathbb{R} \rightarrow \mathbb{R} \text { be such that } \sigma^{2}=b .
$$

Let $\Delta t_{i, j}=\operatorname{Var}\left(\xi_{i, j}\right)$. Let $t_{i, j}$ be given by $t_{i, 0}=t_{0}$ and $t_{i, j+1}=t_{i, j}+\Delta t_{i, j}$. Let stochastic processes $\mathcal{X}_{i}$ recursively be defined by:

$$
\mathcal{X}_{i}\left(t_{0}\right)=x_{0}, \quad \Delta \mathcal{X}_{i}\left(t_{i, j}\right)=a\left(t_{i, j}, \mathcal{X}_{i}\left(t_{i, j}\right)\right) \Delta t_{i, j}+\sigma\left(t_{i, j}, \mathcal{X}_{i}\left(t_{i, j}\right)\right) \xi_{i, j}
$$

and

$$
\mathcal{X}_{i}\left(t_{i, j+1}\right)=\mathcal{X}_{i}\left(t_{i, j}\right)+\Delta \mathcal{X}_{i}\left(t_{i, j}\right)
$$

Suppose further that for any $f \in \mathcal{C}_{0}^{\infty}$ there exists a $\mathcal{C}_{b}^{1,2}$ solution $u^{f}(.,$.$) of the terminal$ value problem (18). Then $t_{i, J_{i}}=T$ independent of $i$ and

$$
u^{f}\left(t_{0}, x_{0}\right)=\lim _{i \rightarrow \infty} \mathbb{E}\left[f \circ \mathcal{X}_{i}(T)\right] .
$$


Proof: That $t_{i, J_{i}}=T$ independent of $i \in \mathbb{N}$ follows from the recursive definition of $t_{i, j}$ and (52).

The theorem stated above is solely stated in the terms of standard mathematics. We can therefore by transfer suppose without loss of generality that any object named in the theorem is standard. Thus again (47) holds. Choose an arbitrary nonstandard $i \in \mathbb{N}$ and let $\left[t_{0} \ldots T\right]:=\left\{t_{i, j} \mid 0 \leq j \leq J_{i}\right\}$. Let for any $t=t_{i, j} \in\left[t_{0} \ldots T\right)$ random variables $\delta W_{t}$ be given by $\delta W_{t}=\xi_{i, j}$ and let $X_{t}:=\mathcal{X}_{i, j}\left(t_{i, j}\right)$. Then $\left(\delta W_{t}\right)_{t \in\left[t_{0} \ldots T\right)}$ is an infinitesimal model of white noise and by (53) the stochastic process $X=\left(X_{t}\right)_{t \in\left[t_{0} \ldots T\right]}$ is the solution of the infinitesimal stochastic difference equation

$$
\delta X_{t}=a\left(t, X_{t}\right) \delta t+\sigma\left(t, X_{t}\right) \delta W_{t} .
$$

with initial condition $X_{t_{0}}=x_{0}$. Application of Theorem 4.13 proves by its conclusion (38) with $u=u^{f}$ that

$$
u^{f}\left(x_{0}, t_{0}\right)=u\left(x_{0}, t_{0}\right) \approx \mathbb{E}\left[f \circ X_{T}\right]=\mathbb{E}\left[f \circ \mathcal{X}_{i}(T)\right]
$$

Since $i$ was assumed to be nonstandard but otherwise arbitrarily chosen we obtain that

$$
\forall^{n s t} i \in \mathbb{N} u^{f}\left(x_{0}, t_{0}\right) \approx \mathbb{E}\left[f \circ \mathcal{X}_{i}(T)\right]
$$

Since by our hypothesis - made at the begin of the proof - that all objects named in the theorem are standard, the sequence $\left(\mathbb{E}\left[f \circ \mathcal{X}_{i}(T)\right]\right)_{i \in \mathbb{N}}$ as well as the real number $u^{f}\left(x_{0}, t_{0}\right)$ are standard. Thus by (55), by Definition 2.4 and Remark 2.5 we obtain that $\lim _{i \rightarrow \infty} \mathbb{E}\left[f \circ \mathcal{X}_{i}\left(t_{i, J_{i}}\right)\right]$ exists and that

$$
u^{f}\left(t_{0}, x_{0}\right)=\lim _{i \rightarrow \infty} \mathbb{E}\left[f \circ \mathcal{X}_{i}(T)\right]
$$

i.e., (54) holds and the theorem has been proved.

Remark 5.5 Suppose that the hypotheses of Theorem 5.4 are fulfilled and that the family of operators $\left(\mathcal{L}_{t}\right)_{t \in\left[t_{0}, T\right]}$ is additionally standard. Then the sequence $\left(\mathcal{X}_{i}(T)\right)_{i \in \mathbb{N}}$ of (by Remark 4.15) almost limited random variables $\mathcal{X}_{i}(T)$ in Theorem 5.4 S-converges approximately to a random variable $\mathcal{X}_{\infty}$ such that for all $f \in \mathcal{C}_{0}^{\infty}$

$$
u^{f}\left(t_{0}, x_{0}\right) \approx \mathbb{E}\left[f \circ \mathcal{X}_{\infty}\right]
$$

Proof: Apply Proposition 3.6 to (55).

Journal of Logic \& Analysis 1:2 (2009) 


\section{An Example}

Remark 6.1 If we suppose that $a(t, x)=0, b(t, x)=1$ and let $T-t_{0}:=\sigma^{2}$, then Theorem 5.4 is closely related to Lindeberg's limit theorem (Theorem 5.2). If we replace hypothesis (43) in Theorem 5.2 by the stronger hypothesis

$$
\forall i \in \mathbb{N} \quad \operatorname{Var}\left(\zeta_{i}\right)=\sigma^{2}
$$

and apply $T-t_{0}=\sigma^{2}$ then we end up with the hypotheses of Theorem 5.4 (in the special case that $a(t, x)=0, b(t, x)=1)$.

The conclusion (54) of Theorem 5.4 implies together with (42), $a(t, x)=0, b(t, x)=1$ and $\sigma^{2}=T-t_{0}$ that (51) holds (if we let $\left.\left(t_{0}, x_{0}\right):=(0,0)\right)$ and thus further that the conclusion of Theorem 5.2 holds.

Thus we conclude that in the case $a(t, x)=0, b(t, x)=1$ and $\sigma^{2}=T-t_{0}$ Theorem 5.4 implies Theorem 5.2 with the hypothesis (43) replaced by (56), i.e., Theorem 5.4 implies a weakened version of Theorem 5.2.

A more elaborate application of Theorem 5.4 for special coefficient functions $a(.,$. and $b(.,$.$) is provided by an approximation of the infinitesimal stochastic difference$ equations (for $\tau>0$ )

$$
\delta X_{t}=-\tanh \left(\tau X_{t}\right)\left(\frac{1}{\tau}+\frac{1}{2} \cdot \frac{\tau}{\cosh ^{2}\left(\tau X_{t}\right)}\right) \delta t+\frac{1}{\cosh \left(\tau X_{t}\right)} \delta W_{t},
$$

with initial condition $X_{t_{0}}=x_{0}$, by the difference equations

$$
\mathcal{X}_{i}(0)=x_{0}, \quad \Delta \mathcal{X}_{i}\left(t_{i, j}\right)=a\left(\mathcal{X}_{i}\left(t_{i, j}\right)\right) \Delta t_{i, j}+\sigma\left(\mathcal{X}_{i}\left(t_{i, j}\right)\right) \xi_{i, j}
$$

with

$$
\begin{gathered}
a(x)=-\tanh (\tau x)\left(\frac{1}{\tau}+\frac{1}{2} \cdot \frac{\tau}{\cosh ^{2}(\tau x)}\right), \\
\sigma(x)=\frac{1}{\cosh (\tau x)}
\end{gathered}
$$

and $\left(\xi_{i, j}\right)_{i, j \in \mathbb{N}}$ a triangular approximation of white noise (Example 6.6 below). We remark that the equations (57) are for small $\tau$ themself approximations of the OrnsteinUhlenbeck infinitesimal stochastic difference equation

$$
\delta X_{t}=-X_{t} \delta t+\delta W_{t} .
$$

Before we state Example 6.6 we prove some results (Proposition 6.2, Remark 6.3, Proposition 6.4 and Corollary 6.5 stated below) that provide together with Theorem 5.4 the right setting for the proof of the validity of Example 6.6. 
Proposition 6.2 Let $g \in \mathcal{C}_{0}^{\infty}$. A $\mathcal{C}_{b}^{1,2}$ solution of the terminal value problem

$$
\frac{1}{2} \cdot \frac{\partial^{2} v(t, y)}{\partial y^{2}}-y \frac{\partial v(t, y)}{\partial y}+\frac{\partial v(t, y)}{\partial t}=0, \quad v(T, y)=g(y)
$$

on the interval $\left[t_{0}, T\right]$ is for $t \in\left[t_{0}, T\right)$ provided by the function

$$
v(t, y)=\int_{z \in \mathbb{R}} \frac{1}{\sqrt{\pi\left(1-e^{-2(T-t)}\right)}} e^{-\frac{\left(z-e^{-(T-t)} y\right)^{2}}{\left(1-e^{-2(T-t)}\right)}} g(z) d z .
$$

Proof: Straightforward calculation shows that (59) is a solution of (58).

Remark 6.3 If $\phi$ denotes the density function of the $N(0,1)$ distribution, then the function $v$ of equation (59) can be expressed as

$$
v(t, y)=\int_{z \in \mathbb{R}} \frac{1}{\sqrt{\frac{1}{2}\left(1-e^{-2(T-t)}\right)}} \phi\left(\frac{z-e^{-(T-t)} y}{\sqrt{\frac{1}{2}\left(1-e^{-2(T-t)}\right)}}\right) g(z) d z .
$$

Consequently, if $Z$ denotes an $N\left(e^{-(T-t)} y, \frac{1}{2}\left(1-e^{-2(T-t)}\right)\right)$ distributed random variable, then $v(t, y) \approx \mathbb{E}[g \circ Z]$.

Proposition 6.4 A function $v:\left[t_{0}, T\right] \times \mathbb{R} \rightarrow \mathbb{R}$ fulfills (58) if and only if $u(t, x)=$ $v\left(t, \frac{1}{\tau} \sinh (\tau x)\right)$ fulfills the terminal value problem

$$
\begin{aligned}
& \frac{\partial u(t, x)}{\partial t}-\tanh (\tau x)\left(\frac{1}{\tau}+\frac{1}{2} \cdot \frac{\tau}{\cosh ^{2}(\tau x)}\right) \frac{\partial u(t, x)}{\partial x}+\frac{1}{2} \cdot \frac{1}{\cosh ^{2}(\tau x)} \frac{\partial^{2} u(t, x)}{\partial x^{2}}=0, \\
& \text { (60) } \quad u(T, x)=g\left(\frac{1}{\tau} \sinh (\tau x)\right) .
\end{aligned}
$$

Thus there exists a one-one correspondence between the solutions of (58) and (60).

Proof: This follows with $y=\frac{1}{\tau} \sinh (\tau x)$ from

$$
\frac{\partial v(t, y)}{\partial t}=\frac{\partial u(t, x)}{\partial t}, \quad \frac{\partial v(t, y)}{\partial y}=\frac{1}{\cosh (\tau x)} \frac{\partial u(t, x)}{\partial x}
$$

and

$$
\frac{1}{2} \cdot \frac{\partial^{2} v(t, y)}{\partial y^{2}}=-\frac{1}{2} \cdot \frac{\tau \tanh (\tau x)}{\cosh ^{2}(\tau x)} \frac{\partial u(t, x)}{\partial x}+\frac{1}{2} \cdot \frac{1}{\cosh ^{2}(\tau x)} \frac{\partial^{2} u(t, x)}{\partial x^{2}} .
$$

Corollary 6.5 There exists a unique $\mathcal{C}_{b}^{1,2}$ solutions of the terminal value problem (60). 
Proof: A solution $v(t, y)$ of (58) exists by Proposition 6.2 and thus by Proposition 6.4 there also exists a solution $u(t, x)=v\left(t, \frac{1}{\tau} \sinh (\tau x)\right)$ of (60). The functions

$$
a(t, x)=-\tanh (\tau x)\left(\frac{1}{\tau}+\frac{1}{2} \cdot \frac{\tau}{\cosh ^{2}(\tau x)}\right) \text { and } b(t, x)=\frac{1}{\cosh ^{2}(\tau x)}
$$

are for any fixed $\tau \in(0, \infty)$ bounded and calculation gives that the solution $u$ of (60) - obtained from the solution (59) of equation (58) by Proposition 6.4 - is an element of $\mathcal{C}_{b}^{1,2}$. Thus we obtain by Corollary 4.9 and transfer that the solution $u(t, x)$ of the terminal value problem (60) is unique.

Example 6.6 Let $\tau \in(0, \infty)$ be standard and let $\Xi$ be a triangular approximation of white noise on $\left[t_{0}, T\right]$. Let $a:\left[t_{0}, T\right] \times \mathbb{R} \rightarrow \mathbb{R}$ and $\sigma:\left[t_{0}, T\right] \times \mathbb{R} \rightarrow \mathbb{R}$ be given by

$$
a(x)=-\tanh (\tau x)\left(\frac{1}{\tau}+\frac{1}{2} \cdot \frac{\tau}{\cosh ^{2}(\tau x)}\right) \text { and } \sigma(x)=\frac{1}{\cosh (\tau x)}
$$

Let $\Delta t_{i, j}=\operatorname{Var}\left(\xi_{i, j}\right)$. Let $t_{i, j}$ be given by $t_{i, 0}=t_{0}$ and $t_{i, j+1}=t_{i, j}+\Delta t_{i, j}$. Let stochastic processes $\mathcal{X}_{i}$ recursively be defined by:

$$
\mathcal{X}_{i}\left(t_{0}\right)=x_{0}, \quad \Delta \mathcal{X}_{i}\left(t_{i, j}\right)=a\left(\mathcal{X}_{i}\left(t_{i, j}\right)\right) \Delta t_{i, j}+\sigma\left(\mathcal{X}_{i}\left(t_{i, j}\right)\right) \xi_{i, j}
$$

and

$$
\mathcal{X}_{i}\left(t_{i, j+1}\right)=\mathcal{X}_{i}\left(t_{i, j}\right)+\Delta \mathcal{X}_{i}\left(t_{i, j}\right)
$$

Then $\left(\mathcal{X}_{i}(T)\right)_{i \in \mathbb{N}}$ S-converges approximately to a random variable $\mathcal{X}_{\infty}$ that fulfills $\mathcal{X}_{\infty}=\frac{1}{\tau} \operatorname{arsinh}(\tau \mathcal{Z})$ for an $N\left(e^{-\left(T-t_{0}\right)} y_{0}, \frac{1}{2}\left(1-e^{-2\left(T-t_{0}\right)}\right)\right)$ distributed random variable $\mathcal{Z}$ with $y_{0}=\frac{1}{\tau} \sinh \left(\tau x_{0}\right)$.

Proof: Note that the hypotheses of Remark 5.5 are fulfilled. Thus by Remark $5.5 \mathcal{X}_{i}(T)$ S-converges approximately to a random variable $\mathcal{X}_{\infty}$. Let $\sinh _{\tau}(x):=\frac{1}{\tau} \sinh (\tau x)$. We conclude from Remark 5.5 (or Theorem 5.4) with $f=g \circ \sinh _{\tau}$, Proposition 6.2, Remark 6.3 and Corollary 6.5 that

$$
\left(\forall^{s t} g \in \mathcal{C}_{0}^{\infty}\right)\left(\begin{array}{c}
\mathbb{E}\left[g \circ \sinh _{\tau} \circ \mathcal{X}_{\infty}\right] \approx u^{g \circ \sinh _{\tau}}\left(t_{0}, x_{0}\right)= \\
v^{g}\left(t_{0}, \frac{1}{\tau} \sinh \left(\tau x_{0}\right)\right)=v^{g}\left(t_{0}, y_{0}\right) \approx \mathbb{E}[g \circ \widetilde{\mathcal{Z}}]
\end{array}\right)
$$

with $\widetilde{\mathcal{Z}}$ an $N\left(e^{-(T-t)} y_{0}, \frac{1}{2}\left(1-e^{-2(T-t)}\right)\right)$ distributed random variable and $y_{0}=\frac{1}{\tau} \sinh \left(\tau x_{0}\right)$. Thus if we let $\mathcal{Z}:=\sinh _{\tau} \circ \mathcal{X}_{\infty}$ then

$$
\forall^{s t} g \in \mathcal{C}_{0}^{\infty} \quad \mathbb{E}[g \circ \mathcal{Z}]=\mathbb{E}\left[g \circ \sinh _{\tau} \circ \mathcal{X}_{\infty}\right] \approx \mathbb{E}[g \circ \widetilde{\mathcal{Z}}]
$$

and thus $\mathcal{Z}$ is also $N\left(e^{-(T-t)} y_{0}, \frac{1}{2}\left(1-e^{-2(T-t)}\right)\right)$ distributed. 


\section{A Appendix}

Definition A.1 Given a function $g: \Omega \rightarrow \mathbb{R}$ we let $g^{(n)}:=g \cdot \mathbb{I}_{\{x \mid-n \leq g(x) \leq n\}}$.

We show that the infinitesimal Lindeberg condition displayed by equation (12) of this article is equivalent with the near Lindeberg condition given by equation (14.1) of [27]. For this purpose we display the near Lindeberg condition (14.1) of [27]:

A family of random variables $\left(\delta W_{t}\right)_{t \in\left[t_{0} \ldots T\right)}$ fulfills the near Lindeberg condition if

$$
\forall^{\mathrm{st}} \varepsilon>0 \mathbb{E}\left[\sum_{t \in\left[t_{0} \ldots T\right)}\left(\delta W_{t}\right)^{2}\right] \approx \mathbb{E}\left[\sum_{t \in\left[t_{0} \ldots T\right)}\left(\delta W_{t}^{(\varepsilon)}\right)^{2}\right] .
$$

Lemma A.2 Suppose that we are given a family of real valued random variables $\left(\delta W_{t}\right)_{t \in\left[t_{0} \ldots T\right)}$ with distributions $\nu_{t}$. Then the infinitesimal Lindeberg condition (12) and the near Lindeberg condition (61) are equivalent.

Proof: We show $(61) \Rightarrow(12)$ first. Formula (61) is, by the linearity of the expectation and the fact that

$$
\left(\delta W_{t}\right)^{2} \geq\left(\delta W_{t}^{(\varepsilon)}\right)^{2}
$$

equivalent with

$$
\forall^{s t} \varepsilon>0 \sum_{t \in\left[t_{0} \ldots T\right)} \mathbb{E}\left[\left(\delta W_{t}\right)^{2}-\left(\delta W_{t}^{(\varepsilon)}\right)^{2}\right]<\varepsilon .
$$

This implies by Proposition 2.3 that

$$
\exists \eta \approx 0 \sum_{t \in\left[t_{0} \ldots T\right)} \mathbb{E}\left[\left(\delta W_{t}\right)^{2}-\left(\delta W_{t}^{(\eta)}\right)^{2}\right]<\eta .
$$

Formula (63) is equivalent with our infinitesimal Lindeberg condition (12). Thus (61) $\Rightarrow(12)$ has been proved and it remains to be shown that (12) implies (61).

Formula (12) is equivalent with (63). Since $\eta \leq \varepsilon$ implies that

$$
\sum_{t \in\left[t_{0} \ldots T\right)} \mathbb{E}\left[\left(\delta W_{t}\right)^{2}-\left(\delta W_{t}^{(\varepsilon)}\right)^{2}\right] \leq \sum_{t \in\left[t_{0} \ldots T\right)} \mathbb{E}\left[\left(\delta W_{t}\right)^{2}-\left(\delta W_{t}^{(\eta)}\right)^{2}\right],
$$

we obtain from (63) and (62) that

$$
\forall^{\mathrm{st}} \varepsilon>0 \sum_{t \in\left[t_{0} \ldots T\right)} \mathbb{E}\left[\left(\delta W_{t}\right)^{2}-\left(\delta W_{t}^{(\varepsilon)}\right)^{2}\right] \approx 0 .
$$


Formula (64) implies by the linearity of the expectation that (61) holds. Thus (12) $\Rightarrow$ (61) has been established and the lemma has been proved.

There exists a further condition (65) that - although seemingly weaker than (61) - turns out to be equivalent with (61). To state this condition we need the following definition:

Definition A.3 Given a function $g: \Omega \rightarrow \mathbb{R}$ we let $\left.g\right|^{n}:=\max (\min (g, n),-n)$.

Lemma A.4 Suppose that we are given a family of real valued random variables $\left(\delta W_{t}\right)_{t \in\left[t_{0} \ldots T\right)}$ with distributions $\nu_{t}$. Then the equivalent conditions (12) and (61) are further equivalent with

$$
\forall s t_{\varepsilon}>0 \mathbb{E}\left[\sum_{t \in\left[t_{0} \ldots T\right)}\left(\delta W_{t}\right)^{2}\right] \approx \mathbb{E}\left[\sum_{t \in\left[t_{0} \ldots T\right)}\left(\left.\delta W_{t}\right|^{\varepsilon}\right)^{2}\right] .
$$

Proof: We show $(61) \Rightarrow(65) \Rightarrow(12)$. Since

$$
\sum_{t \in\left[t_{0} \ldots T\right)}\left(\delta W_{t}\right)^{2} \geq \sum_{t \in\left[t_{0} \ldots T\right)}\left(\left.\delta W_{t}\right|^{\varepsilon}\right)^{2} \geq \sum_{t \in\left[t_{0} \ldots T\right)}\left(\delta W_{t}^{(\varepsilon)}\right)^{2},
$$

we obtain that $(61) \Rightarrow(65)$ holds and it remains to be show that $(65) \Rightarrow(12)$. Formula (65) is, by the linearity of the expectation and the fact that

$$
\left(\delta W_{t}\right)^{2} \geq\left(\left.\delta W_{t}\right|^{\varepsilon}\right)^{2},
$$

equivalent with

$$
\forall^{s t} \varepsilon>0 \sum_{t \in\left[t_{0} \ldots T\right)} \mathbb{E}\left[\left(\delta W_{t}\right)^{2}-\left(\left.\delta W_{t}\right|^{\varepsilon}\right)^{2}\right]<\varepsilon .
$$

This implies by Proposition 2.3 that

$$
\exists \hat{\eta} \approx 0 \sum_{t \in\left[t_{0} \ldots T\right)} \mathbb{E}\left[\left(\delta W_{t}\right)^{2}-\left(\left.\delta W_{t}\right|^{\hat{\eta}}\right)^{2}\right]<\hat{\eta} .
$$

Formula (66) is equivalent with

$$
\exists \hat{\eta} \approx 0 \sum_{t \in\left[t_{0} \ldots T\right)}\left[\int_{|y| \geq \hat{\eta}}\left(y^{2}-\hat{\eta}^{2}\right) d \nu_{t}(y)\right]<\hat{\eta} .
$$

Formula (67) implies that

(68) $\exists \hat{\eta} \approx 0 \sum_{t \in\left[t_{0} \ldots T\right)}\left[\int_{|y| \geq 2 \hat{\eta}} \hat{\eta}^{2} d \nu_{t}(y)\right]<\sum_{t \in\left[t_{0} \ldots T\right)}\left[\int_{|y| \geq 2 \hat{\eta}}\left(y^{2}-\hat{\eta}^{2}\right) d \nu_{t}(y)\right]<\hat{\eta}$. 
From (68) we obtain that

$$
\begin{aligned}
\exists \hat{\eta} \approx 0 & \sum_{t \in\left[t_{0} \ldots T\right)}\left[\int_{|y| \geq 2 \hat{\eta}} y^{2} d \nu_{t}(y)\right]= \\
& \sum_{t \in\left[t_{0} \ldots T\right)}\left[\int_{|y| \geq 2 \hat{\eta}}\left(y^{2}-\hat{\eta}^{2}\right) d \nu_{t}(y)\right]+\sum_{t \in\left[t_{0} \ldots T\right)}\left[\int_{|y| \geq 2 \hat{\eta}} \hat{\eta}^{2} d \nu_{t}(y)\right]<2 \hat{\eta} .
\end{aligned}
$$

Letting $\eta=2 \hat{\eta}$ we finally obtain (12) from (69). Thus (65) $\Rightarrow(12)$ has been proved.

Acknowledgement: I would like to thank the following persons for discussion either concerning the content of this paper or techniques in- and the logical and philosophical background of nonstandard analysis, that inspired me and helped me to write the paper: R. M. Anderson, E. Benoît, I. Van den Berg, N. Cutland, E. Dolejsi, H. Ploss, G. Pflug, M. Reeken, D. Schwabel.

I would further like to thank the referees for their excellent work; especially I would like to thank them for pointing out a serious mistake in a proof of a previously submitted (November 2006) and rejected precursor of this article. I would also like to thank I. Van den Berg for pointing out the same mistake to me.

\section{References}

[1] Anderson, Robert M. A nonstandard representation for Brownian motion and Ito integration. Isr. J. Math., 25, 15-46 (1976); doi:10.1007/BF02756559

[2] Anderson, Robert M.; Rashid, Salim A nonstandard characterization of weak convergence. Proc. Am. Math. Soc. 69, 327-332 (1978); doi:10.2307/2042621.

[3] Benoît, E. Random walks and stochastic differential equations. Nonstandard analysis in practice. Diener, Francine (ed.); Diener, Marc (ed.) Universitext. Berlin: SpringerVerlag. (1995).

[4] Van den Berg, I. Equations paraboliques et intégrales de chemins finies avec applications financières. http://math1.unice.fr/ rgr/prepubli.html

[5] Van den Berg, I. Principles of infinitesimal stochastic and financial analysis. Singapore: World Scientific. (2000).

[6] Capiński, M.; Cutland, N. Statistical solutions of stochastic Navier-Stokes equations. Indiana Univ. Math. J. 43, No.3, 927-940 (1994). doi:10.1512/iumj.1994.43.43040. 
[7] Capiński, M.; Cutland, N. Foiaş and Hopf statistical solutions for stochastic NavierStokes equations. Stochastics and Stochastics Rep. 52, No.3-4, 193-205 (1995).

[8] Cutland, N.J. Loeb measures in practice: Recent advances. EMS lectures 1997. Lecture Notes in Mathematics. 1751. Berlin: Springer. (2000).

[9] Cutland, N.J.; Ng, S.-A. A nonstandard approach to the Malliavin calculus in Albeverio, Sergio A. (ed.) et al. Advances in analysis, probability and mathematical physics: contributions of nonstandard analysis. Kluwer Academic Publishers. Math. Appl., Dordr. 314, 149-170 (1995).

[10] Diener, F; Diener M. Tutorial. Nonstandard analysis in practice. Diener, Francine (ed.); Diener, Marc (ed.) Universitext. Berlin: Springer-Verlag. (1995).

[11] Dudley, R.M. Real analysis and probability. Repr. Cambridge Studies in Advanced Mathematics. 74. Cambridge: Cambridge University Press, 2002.

[12] Fajardo, S.; Keisler, J. Model theory of stochastic processes. Lecture Notes in Logic. 14. Urbana, IL: Association for Symbolic Logic. Natick, MA: A K Peters, (2002).

[13] Friedman, A. Partial differential equations of parabolic type. Englewood Cliffs, N.J.: Prentice-Hall, Inc. (1964).

[14] Gard, T. Introduction to stochastic differential equations. Pure and Applied Mathematics, Marcel Dekker 114. New York etc.: Marcel Dekker, Inc. (1988).

[15] Kanovei, V.; Reeken, M. Nonstandard analysis, axiomatically. Springer Monographs in Mathematics. Berlin: Springer. (2004).

[16] Karatzas, I.; Shreve, S. Brownian motion and stochastic calculus. 2nd ed. Graduate Texts in Mathematics, 113. New York etc.: Springer-Verlag. (1991).

[17] Keisler, H.J. Stochastic differential equations with extra properties, in Arkeryd, Leif O. (ed.) et al., Nonstandard analysis: theory and applications. Kluwer Academic Publishers. NATO ASI Ser., Ser. C, Math. Phys. Sci. 493, 259-277 (1997).

[18] Kloeden, P.; Platen, E. Numerical solution of stochastic differential equations. Applications of Mathematics. 23. Berlin: Springer-Verlag. (1992).

[19] Koudjeti F., Van den Berg I. Neutrices, external numbers, and external calculus. Nonstandard analysis in practice. Diener, Francine (ed.); Diener, Marc (ed.) Universitext. Berlin: Springer-Verlag. (1995).

[20] Lapeyre, B.; Pardoux, E.; Sentis, R. Introduction to Monte-Carlo methods for transport and diffusion equations. Translated from the French by Alain Craig and Fionn Craig. Oxford Texts in Applied and Engineering Mathematics 6. Oxford: Oxford University Press. (2003).

[21] Lindstrøm, T. An invitation to nonstandard analysis. (English) Nonstandard analysis and its applications, Pap. Conf., Hull/UK 1986, Lond. Math. Soc. Stud. Texts 10, 1-105 (1988).

[22] Lindstrøm, T. Hyperfinite stochastic integration. I, II, III Math. Scand. 46, 265-292, 293-314, 315-331 (1980). 
[23] Lindstrøm, T. Anderson's Brownian motion and the infinite dimensional OrnsteinUhlenbeck process, in Albeverio, Sergio A. (ed.) et al. Advances in analysis, probability and mathematical physics: contributions of nonstandard analysis, Kluwer Academic Publishers. Math. Appl., Dordr. 314, 186-199 (1995).

[24] Lindstrøm, T. Internal martingales and stochastic integration in Arkeryd, Leif O. (ed.) et al. Nonstandard analysis: theory and applications. Kluwer Academic Publishers. NATO ASI Ser., Ser. C, Math. Phys. Sci. 493, 209-258 (1997).

[25] Loeb, Peter A. Conversion from nonstandard to standard measure spaces and applications in probability theory. Trans. Am. Math. Soc. 211, 113-122 (1975); doi:10.2307/1997222

[26] Nelson, E. Internal set theory: A new approach to nonstandard analysis. Bull. Am. Math. Soc. 83, 1165-1198 (1977); doi:10.1090/S0002-9904-1977-14398-X

[27] Nelson, E. Radically elementary probability theory. Annals of Mathematics Studies, No.117. Princeton, New Jersey: Princeton University Press. (1987).

[28] Oeksendal, B. Stochastic differential equations. An introduction with applications. 6th $e d$. Universitext. Berlin: Springer. (2003).

[29] Robinson, A. Non-standard analysis. Repr. Princeton, NJ: Princeton Univ. Press. (1996).

[30] Schervish, Mark J. Theory of statistics. Springer Series in Statistics. New York, NY: Springer-Verlag. (1995).

[31] Schuss, Z. Theory and applications of stochastic differential equations. Wiley Series in Probability and Mathematical Statistics. New York etc.: John Wiley \& Sons. (1980).

[32] Sobczyk, K. Stochastic differential equations. With applications to physics and engineering. Mathematics and Its Applications. East European Series. 40. Dordrecht etc.: Kluwer Academic Publishers. (1991).

[33] Steele, J.M. Stochastic calculus and financial applications. Applications of Mathematics. 45. New York, NY: Springer.

[34] Stroock, D.; Varadhan, S. Multidimensional diffusion processes. Grundlehren der mathematischen Wissenschaften. 233. Berlin, Heidelberg, New York: Springer-Verlag. (1979).

[35] Stroyan, K.; Bayod, J. Foundations of infinitesimal stochastic analysis. Studies in Logic and the Foundations of Mathematics, Vol. 119. Amsterdam- New York-Oxford: North-Holland. (1986).

ZBSA University of Freiburg, Habsburgerstrasse 49, 79104 Freiburg, Germany

heinz. weisshaupt@zbsa.uni-freiburg. de,

heinz.weisshaupt@univie.ac . at

Received: 28 October 2007 